\title{
Selective Peroxisome Proliferator-Activated Receptor Alpha Modulators (SPPARMa) in the Metabolic Syndrome: Is Pemafibrate Light at the End of the Tunnel?
}

\author{
Jean-Charles Fruchart ${ }^{1} \cdot$ Michel P. Hermans $^{2} \cdot$ Jamila Fruchart-Najib $^{1} \cdot$ Tatsuhiko Kodama $^{3}$ \\ Accepted: 11 November 2020 / Published online: 3 January 2021 \\ (C) The Author(s) 2021
}

\begin{abstract}
Purpose of Review Adoption of poor lifestyles (inactivity and energy-dense diets) has driven the worldwide increase in the metabolic syndrome, type 2 diabetes mellitus and non-alcoholic steatohepatitis (NASH). Of the defining features of the metabolic syndrome, an atherogenic dyslipidaemia characterised by elevated triglycerides (TG) and low plasma concentration of highdensity lipoprotein cholesterol is a major driver of risk for atherosclerotic cardiovascular disease. Beyond lifestyle intervention and statins, targeting the nuclear receptor peroxisome proliferator-activated receptor alpha (PPAR $\alpha$ ) is a therapeutic option. However, current PPAR $\alpha$ agonists (fibrates) have limitations, including safety issues and the lack of definitive evidence for cardiovascular benefit. Modulating the ligand structure to enhance binding at the PPAR $\alpha$ receptor, with the aim of maximising beneficial effects and minimising adverse effects, underlies the SPPARM $\alpha$ concept.

Recent Findings This review discusses the history of SPPARM development, latterly focusing on evidence for the first licensed SPPARM $\alpha$, pemafibrate. Evidence from animal models of hypertriglyceridaemia or NASH, as well as clinical trials in patients with atherogenic dyslipidaemia, are overviewed.

Summary The available data set the scene for therapeutic application of SPPARM $\alpha$ in the metabolic syndrome, and possibly, NASH. The outstanding question, which has so far eluded fibrates in the setting of current evidence-based therapy including statins, is whether treatment with pemafibrate significantly reduces cardiovascular events in patients with atherogenic dyslipidaemia. The PROMINENT study in patients with type 2 diabetes mellitus and this dyslipidaemia is critical to evaluating this.
\end{abstract}

Keywords Pemafibrate Selective peroxisome proliferator-activated receptor alpha modulator $\cdot$ SPPARM $\cdot$ Triglycerides . Metabolic syndrome $\cdot$ Non-alcoholic fatty liver disease

\section{Introduction}

The metabolic syndrome poses a global challenge as societies become increasingly urbanised, sedentary and obese. A key requirement for identification is the

This article is part of the Topical Collection on Statin Drugs

Jean-Charles Fruchart

Jean-Charles.fruchart@r3i.org

Michel P. Hermans

michel.hermans@uclouvain.be

Jamila Fruchart-Najib

jamila.fruchart@yahoo.fr

Tatsuhiko Kodama

kodama@1sbm.org combination of three or more of the following: increased waist circumference, elevated triglycerides (TG), low plasma concentration of high-density lipoprotein cholesterol (HDL-C), elevated blood pressure and raised fasting blood glucose (Table 1) [1]. Worldwide, $20-30 \%$ of

1 Residual Risk Reduction Initiative (R3i) Foundation, Picassoplatz 8, 4010 Basel, Switzerland

2 Division of Endocrinology and Nutrition, Cliniques Universitaires St-Luc and Institut de Recherche Expérimentale et Clinique (IREC), Université Catholique de Louvain, Brussels, Belgium

3 Laboratory for System Biology and Medicine. Research Center for Advanced Science and Technology, The University of Tokyo, Tokyo, Japan 
Table 1 Harmonised definition of the metabolic syndrome. Derived from Alberti et al [1]

Waist $>94 \mathrm{~cm}$ (men) or $>80 \mathrm{~cm}$ (women)* together with the presence of two or more of the following:

Fasting blood glucose greater than $5.6 \mathrm{mmol} / \mathrm{L}(100 \mathrm{mg} / \mathrm{dL})$ or diagnosed diabetes

HDL cholesterol $<1.0 \mathrm{mmol} / \mathrm{L}(40 \mathrm{mg} / \mathrm{dL})$ in men, $<1.3 \mathrm{mmol} / \mathrm{L}$ $(50 \mathrm{mg} / \mathrm{dL})$ in women or drug treatment for low HDL-C

Fasting blood triglycerides $>1.7 \mathrm{mmol} / \mathrm{L}(150 \mathrm{mg} / \mathrm{dL})$ or drug treatment for elevated triglycerides

Blood pressure $>130 / 85 \mathrm{mmHg}$ or drug treatment for hypertension

*Based on the International Diabetes Federation thresholds for Europid population, with subsequent regional-specific definitions in men and women

adults are affected, although this varies with age, ethnicity and gender [2]. The metabolic syndrome is no longer a disease of affluence, with escalating prevalence in emerging regions coincident with rising rates of obesity [3, 4], or a disease of adults, as globally more than $5 \%$ of children and adolescents are affected [4, 5]. The extensive comorbidity of the metabolic syndrome confers a substantial burden, from the increased risk for atherosclerotic cardiovascular disease (ASCVD), affecting multiple vascular territories [6], and type 2 diabetes mellitus [7, 8]. In fact, ASCVD and diabetes are the two leading causes of death.

Beyond the increased risk for ASCVD, metabolic derangements that characterise the metabolic syndrome predispose to the development of non-alcoholic fatty liver disease (NAFLD), the most common chronic liver disease worldwide. NAFLD encompasses the spectrum of disease ranging from asymptomatic fatty infiltration of hepatocytes in the absence of inflammation to progression to non-alcoholic steatohepatitis (NASH), liver fibrosis and liver failure. Among NAFLD patients, about half exhibit the metabolic syndrome with dyslipidaemia the most prevalent characteristic (in $\sim 70 \%$ of patients) [9]. The pathogenesis of NAFLD is multifactorial, involving genetic, environmental and metabolic factors. Of the latter, TG accumulation in the liver, reflecting the imbalance between free fatty acid influx and efflux/catabolism, is a hallmark feature which also drives inflammation $[10,11]$. Globally, it is estimated that NAFLD affects about one billion people, with an overall prevalence of $\sim 25 \%$ in adults, with the highest rates in South America, the Middle East and Asia [9]. The clinical, economic and health-related quality-of-life burden of NAFLD is already substantial and growing [9].

Thus, given the changing landscape of cardiovascular risk associated with escalating obesity, the metabolic syndrome poses a global socioeconomic challenge. Renewed thinking about therapeutic options is imperative.

\section{Pathogenesis}

A key driver of the metabolic syndrome is visceral obesity, a marker of ectopic fat deposition [12•, 13•] (Fig. 1). Expansion of visceral adipose tissue to a greater extent than that of subcutaneous adipose tissue is associated with metabolic alterations which promote inflammation. Key amongst these metabolic derangements is atherogenic dyslipidaemia, in particular increases in TG-rich lipoproteins, their remnants and apolipoprotein (apo) C-III $[14,15]$. These effects are mediated via crosstalk between a multitude of pathways that promote impaired adipogenesis, adipokine dysregulation and inflammation, and increase free fatty acids, oxidative stress, adipose tissue hypoxia and lipotoxicity (both local and systemic) [16••]. Recently, attention has focused on angiopoietin-like protein 2 (ANGPTL2), a glycoprotein which is expressed abundantly in adipose tissue. Under normal conditions, ANGPTL2-mediated expression contributes to angiogenesis and tissue damage repair, whereas overexpression promotes chronic inflammation [17-19]. In obese women with insulin resistance, ANGPTL2 production by adipocytes was shown to upregulate proinflammatory cytokine production in macrophages, in turn increasing adipose tissue inflammation, systemic insulin resistance and hyperinsulinaemia [20]. Thus, ANGPTL2 provides a link between the metabolic syndrome, NAFLD and ASCVD.

Hypertriglyceridaemia is a key component of the dyslipidaemia associated with the metabolic syndrome and NAFLD, its hepatic manifestation. Moderately elevated TG levels (a surrogate for elevated TG-rich lipoproteins and their remnants) result from increased dietary-derived apo B48containing intestinal chylomicrons, overproduction of hepatic very low-density lipoproteins (VLDL) and reduction in catabolism of TG-rich lipoproteins. Evidence from epidemiologic, mechanistic and genetic studies supports a causal association between TG-rich lipoproteins and their TG-hydrolysed remnants and ASCVD [21・,22]. Specifically, it is the cholesterol contained within these lipoproteins (i.e. remnant cholesterol) that promotes the development of atherosclerosis and ischaemic heart disease, in part mediated via low-grade inflammation [23, 24]. Postprandial hypertriglyceridaemia is also an emerging contributing factor in residual cardiovascular risk [25•]. Clinically, the combination of elevated TG and increased waist circumference (hypertriglyceridaemic waist) represents a marker of high-risk carotid atherosclerosis features, and highlighting this dual anomaly can improve the identification of individuals with metabolic syndrome and preclinical atherosclerosis beyond traditional risk factors $\left[26^{\bullet}\right]$.

\section{PPAR: a Key Therapeutic Target}

Lifestyle intervention, encompassing both dietary changes and increased physical activity, is an important first step in the 




Fig. 1 Dyslipidaemia is an important feature of the metabolic syndrome. Overproduction of large very low-density lipoprotein particles is a fundamental defect contributing to the increase in the triglyceride pool. This initiates a sequence of lipoprotein changes, leading to higher levels

management of mild to moderate hypertriglyceridaemia, but longterm adherence is usually problematic [27]. Beyond lifestyle, agents that target the nuclear receptor peroxisome proliferatoractivated receptor (PPAR) are obvious therapeutic candidates given their role in regulating the expression of key genes involved in adipogenesis, lipoprotein metabolism, inflammation and metabolic homeostasis $[28 \bullet \bullet, 29,30]$. Three isoforms are identified to date, $\operatorname{PPAR} \alpha, \operatorname{PPAR} \gamma$ and PPAR $\beta / \delta$, each encoded by separate genes [31]. PPAR $\alpha$ is abundant in energy-demanding tissues, such as the liver, kidney, heart and skeletal muscle; PPAR $\gamma$ is predominantly found in adipose tissue, macrophages and the large intestine, whereas PPAR $\beta / \delta$ is more ubiquitous in distribution $[32,33]$. These PPARs are controlled through their interaction with fatty acids and their derivatives and are the pharmacological targets for the lipid-lowering fibrates (PPAR $\alpha$ ) or the insulin sensitizer thiazolidinediones (PPAR $\gamma$ ).

\section{PPARa}

Activation of PPAR $\alpha$ by binding of endogenous ligands (e.g. fatty acids or eicosanoids), or drugs (fibrates) to the ligand binding domain and subsequent heterodimerisation with the ligandactivated retinoid X-receptor (RXR) trigger a conformational change which influences cofactor recruitment, either promoting (transactivation) or inhibiting (transrepression) expression of target genes. This process is mediated by the interaction between the activated PPAR $\alpha$, the PPAR response element (PPRE) of the target gene and relevant cofactors which render the complex transcriptionally active (or inactive in the case of transrepression) [32]. PPAR $\alpha$ activation targets key genes involved in TG metabolism, specifically increasing the production of lipoprotein lipase and apo $\mathrm{A}-\mathrm{V}$ and decreasing plasma levels of apo C-III; increasing HDL synthesis by targeting genes encoding apo A-I and A-II, scavenger of remnant particles, an increase in small, dense low-density lipoprotein particles and lower plasma concentration of high-density lipoprotein cholesterol. IDL, intermediate-density lipoprotein; HDL-C, high-density lipoprotein cholesterol; LDL, low-density lipoprotein; TG, triglyceride

receptor $\mathrm{BI}$, and the ATP binding cassette transporters $\mathrm{A} 1$ and $\mathrm{G} 1$; and enhancing beta-oxidation by increasing expression of hepatic acyl CoA synthase [34-38]. The net effects are reduction in serum TG, an increase in HDL-C concentration, attenuation of very-lowdensity lipoprotein (VLDL) particles, as well as a shift in the lowdensity lipoprotein (LDL) profile to fewer small, dense LDL particles and a proportional increase in larger, less dense LDL particles. There is also transrepression of proinflammatory genes, leading to lower levels of inflammatory mediators such as C-reactive protein, interleukin-6 and prostaglandins [39]. Emerging evidence also suggests that PPAR $\alpha$ favourably influences glucose homeostasis and insulin sensitivity, possibly mediated via effects on acetyl-CoA [40], and inhibits thrombogenesis and improves vascular function, although the underlying mechanisms are not fully defined.

\section{Other PPAR Isoforms}

PPAR $\gamma$ appears to be important in cell differentiation and energy metabolism, binding to the PPRE of almost all adipogenic genes, including those implicated in glucose and fatty acid metabolism. Although less well characterised, PPAR $\beta / \delta$ appears to regulate lipid metabolism, glucose homeostasis and inflammation, suggesting a role in the maintenance of energy homeostasis [29, 41].

\section{Current Therapeutic Options}

\section{PPARa}

Given their pharmacological profile, PPAR $\alpha$ ligands (fibrates) are appropriate treatments for correcting atherogenic 
dyslipidaemia that is characteristic of the metabolic syndrome. Current fibrates are either specific for PPAR $\alpha$ (fenofibrate and gemfibrozil) or activate all three PPAR subtypes (pan-agonist, bezafibrate). While the TG-lowering efficacy of fibrates is well established, their clinical benefit in terms of reduction in cardiovascular events is less convincing. Two prospective trials with gemfibrozil, the Helsinki Heart Study, a primary prevention trial in men with elevated non-HDL-C [42], and the Veterans Affairs High-Density Lipoprotein Cholesterol Intervention Trial (VA-HIT), a secondary prevention trial in men with low HDL-C [43], showed significant reduction in cardiovascular events. It must be borne in mind, however, that both were essentially monotherapy lipid-lowering trials as these were conducted before widespread statin use. Of the remaining trials, two with fenofibrate, Fenofibrate Intervention and Event Lowering in Diabetes (FIELD) [44] and the Action to Control Cardiovascular Risk in Diabetes (ACCORD) Lipid trial [45], both in patients with type 2 diabetes, and one with bezafibrate (Bezafibrate Infarction Prevention [BIP] study) in patients with established coronary disease [46] were inconclusive. None was positive in terms of reduction in their primary endpoint for the total study population, and in the case of the FIELD study, was further complicated by discrepancies in the uptake of statin use between the two groups. The ACCORD Lipid study was the only trial conducted against background statin treatment; the lack of benefit may largely relate to inappropriate patient selection in terms of baseline TG levels and prevalence of atherogenic dyslipidaemia at inclusion [45].

There are, however, important insights from subgroup analyse of the fibrate trials. Analyses of the Helsinki Heart Study and the VA-HIT showed greater reduction in cardiovascular events in patients with the combination of both elevated TG and low HDL-C [47], or with insulin resistance [48], respectively. In the FIELD study, post hoc analysis showed that patients who satisfied the metabolic syndrome criteria (about $80 \%$ of the total study population), derived greater clinical benefit, with maximum reduction in cardiovascular events in those with the combination of elevated TG and low HDL-C [49]. Subgroup analysis of the ACCORD Lipid study also showed benefit in type 2 diabetes patients with this atherogenic dyslipidaemia [45]. When data from the major fibrate trials were combined, individuals with atherogenic dyslipidaemia gained significant clinical benefit for reduction in risk of cardiovascular events, whereas those without this lipid profile did not [50].

Longer-term follow-up of the BIP study showed that patients with the combination of three of the five criteria of the metabolic syndrome derived significant benefit in terms of reduction in myocardial infarction [51]; added to this, 22year follow-up also showed that elevated TG was a significant predictor of all-cause mortality [52]. There is also evidence to suggest a legacy cardiovascular benefit from fenofibrate treatment in the ACCORDION study, an observational follow-up of the ACCORD Lipid study [53].

There are, however, well-recognised safety concerns with the current fibrates. A major issue is an elevation in serum creatinine with fenofibrate [54]; although this is reversible, there are practical disadvantages in stopping and restarting treatment, as well as limitations to its use in patients with renal dysfunction. The potential for drug-drug interactions is another issue, most notably the risk of myopathy with statin coadministration, clearly demonstrated with gemfibrozil $[55,56]$.

\section{Other PPAR Agonists}

PPAR $\gamma$ agonists are currently limited to pioglitazone, indicated as a glucose-lowering agent for the management of type 2 diabetes mellitus [57]. In addition to beneficial effects on atherogenic dyslipidaemia, pioglitazone has been shown to regress atherosclerosis and reduce cardiovascular events in this patient group [58-60]. The IRIS (Insulin Resistance Intervention after Stroke) trial demonstrated cardiovascular benefit in patients with insulin resistance but without diabetes [61], and significantly reduced the development of diabetes [62]. Safety issues in the trial included weight gain and increases in fracture risk and oedema [63].

Despite encouraging experimental findings, the clinical development of PPAR $\beta / \delta$ agonists in metabolic disorders has been disappointing. Seladelpar (MBX-8025) favourably impacted metabolic parameters, reducing apo B100, TG, nonHDL-C and C-reactive protein and increasing HDL-C, in a short-term trial in overweight men and women with mixed dyslipidaemia, with and without atorvastatin treatment [64]. There was, however, no benefit in NASH, leading to termination of clinical development of this agent [65].

Improved understanding of interactions at the PPAR receptor has invigorated the search for selective and potent peroxisome proliferator-activated modulators (SPPARMs), which aim to maximise beneficial effects and minimise the adverse effects of current PPAR agonists [28••].

\section{SPPARMs for the Metabolic Syndrome?}

The underlying aim of a SPPARM is to improve specificity and potency (i.e. efficacy) and minimise safety issues with established PPAR agonists, such as fibrates. This rationale borrows from that used in the development of selective oestrogen receptor modulators [66]. Understanding binding interactions at the PPAR has been key to the development of SPPARMs, as previously discussed [28••]. In brief, binding of the ligand (drug) at the receptor induces specific conformational changes and selective recruitment of coactivators which then selectively activate or repress key target genes, with 
downstream therapeutic effects. This is the paradigm on which the search for a SPPARM is based.

In the history of SPPARM development, some have shown dual activity, such as aleglitazar, a PPAR $\alpha / \gamma$ agonist (terminated) and elafibranor (previously known as GFT505), a PPAR $\alpha / \delta$ agonist, which had been targeted to the management of NASH. The latest interim analysis of the RESOLVE-IT Phase 3 trial with elafibranor, however, failed to show a significant benefit for the predefined primary endpoint of NASH resolution without worsening of fibrosis, or secondary endpoints related to metabolic parameters versus placebo [67]. Most recently, lanifibranor, a pan-PPAR agonist, met primary and secondary endpoints in a phase $2 b$ study in NASH $[68,69]$.

The very few pure SPPARM $\alpha$ agonists that have been developed to date include LY518674, GW7647 and most recently, pemafibrate (previously referred to as K-877), now licensed in Japan for the management of dyslipidaemia [70]. LY518674 potently upregulated apo A-I production and catabolism in human subjects with the metabolic syndrome [71] but was not superior to fenofibrate in lowering TG and raising HDL-C in patients with atherogenic dyslipidaemia. Additionally, elevation in serum creatinine (similar to that observed with fenofibrate) was also reported with this agent [72].

Pemafibrate is the culmination of the systematic synthesis of over 1500 compounds which were rigorously screened for SPPARM $\alpha$ activity. As for traditional PPAR $\alpha$ agonists, the pemafibrate molecule has an acidic region, but the addition of unique benzoxazole and phenoxyalkyl sidechains gives it a Yshaped structure. As a result, pemafibrate has an enhanced fit within the ligand-binding domain of the PPAR $\alpha$ (Fig. 2) [73, $74,75 \cdot$. This structural differentiation confers an increase in PPAR $\alpha$ activation potency compared with other fibrates and a high degree of PPAR $\alpha$ subtype selectivity [28, 76]. Transcriptome analysis showed that gene expression profiles also differed between these two agents, particularly in terms of magnitude of effect. For example, pemafibrate induced key target genes such as $V L D L R$ and $A B C A 1$ at a concentration 10-fold lower than fenofibrate [77].

In preclinical studies, there was more robust reduction in TG and elevation in HDL-C with pemafibrate compared with fenofibrate, as well as enhanced cholesterol efflux from macrophages, upregulation of fibroblast growth factor 21 (FGF21 ), reduced inflammation and attenuation of atherosclerosis [78]. Additionally, pemafibrate attenuated postprandial hypertriglyceridaemia in a mouse model [79], by suppressing the postprandial increase in chylomicrons and the accumulation of chylomicron remnants. This response was achieved with a pemafibrate dose 100 -fold lower than with fenofibrate [79], implying that pemafibrate is more efficient in decreasing TG and apo B48-containing chylomicron remnants, which are highly atherogenic lipoproteins, more so than those containing apoB100 [80, 81].

Insights from rodent models of NASH have suggested that pemafibrate may have potential in NASH. In a diet-induced amylin NASH mouse model, pemafibrate improved
Fig. 2 Pemafibrate is the realisation of the SPPARM $\alpha$ concept. Understanding binding interactions at the PPAR have been critical in driving the development of SPPARM $\alpha$. Systematic structural modifications based on a precision medicine approach led to the creation of pemafibrate. This agent demonstrated an enhanced fit completely within the ligand binding domain of $\operatorname{PPAR} \alpha$, in contrast to the linear structure of conventional fibrates such as fenofibrate. PPAR $\alpha$, peroxisome proliferator-activated receptor alpha

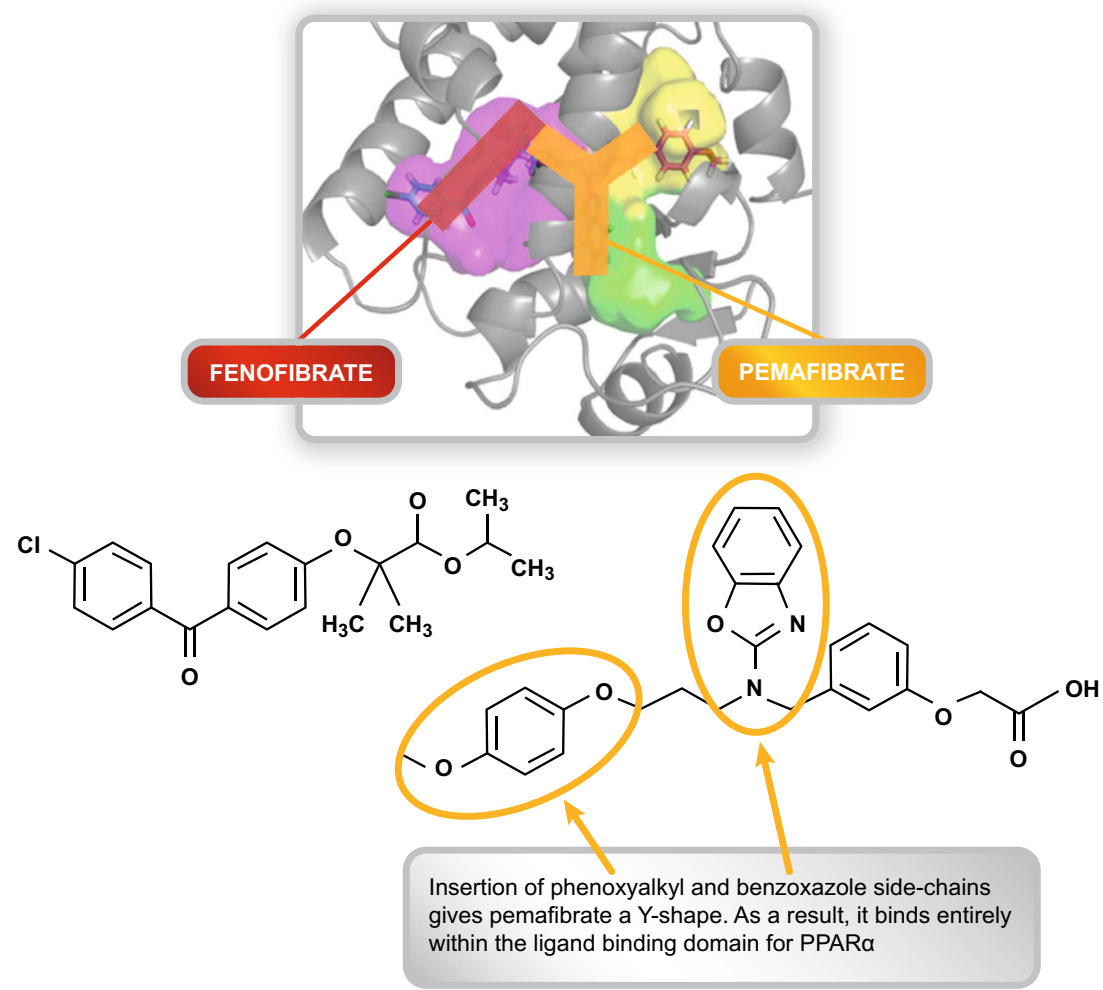


dyslipidaemia, liver dysfunction and NASH features. These effects were attributed in part to stimulation of lipid turnover [82•]. A subsequent study using a STAM NASH mouse model which demonstrates NASH progression resembling the clinical disease showed that pemafibrate improved the histological severity of $\mathrm{NASH}$, as well as inflammatory and fibrosis marker gene expression, without influencing hepatic TG content [83•]. The findings from this study are therefore consistent with current thinking that combination therapy targeting multiple components is needed to manage NASH [84].

\section{Pemafibrate in Metabolic Syndrome: Clinical Profiling}

Preclinical data support the SPPARM $\alpha$ concept and suggest potential therapeutic application in managing dyslipidaemia associated with the metabolic syndrome. Additionally, because pemafibrate is metabolised in the liver and excreted into the bile [85], it can be used safely in patients with renal impairment, as borne out in clinical trials $[86,87 \bullet]$.

An early study in Japanese subjects with atherogenic dyslipidaemia (TG > $200 \mathrm{mg} / \mathrm{dL}$ and low HDL-C, $<50 \mathrm{mg} / \mathrm{dL}$ in men and $<55 \mathrm{mg} / \mathrm{dL}$ in women) showed robust TGlowering (by $\sim 45 \%$ ) with pemafibrate $(0.2$ or $0.4 \mathrm{mg}$ daily) that was superior to fenofibrate $100 \mathrm{mg}$ daily [88]. Pemafibrate treatment was also effective against other components of the dyslipidaemia associated with the metabolic syndrome, including lowering VLDL-C (43 to 48\%), remnant cholesterol (48 to 50\%) and apo B and C-III, raising HDL-C (21 to 14\%) and promoting a shift to a more favourable lipoprotein profile, with fewer small and very small LDL. Importantly, pemafibrate treatment was well tolerated with no increase in serum creatinine and decreased liver enzymes [88]. Pemafibrate also attenuated postprandial hyperlipidaemia [89], consistent with preclinical findings [90] and reduced inflammatory markers such as serum amyloid A and high-sensitivity C-reactive protein [89].

Furthermore, pemafibrate $(0.4 \mathrm{mg}$ daily for 24 weeks) was similarly effective in Japanese patients with type 2 diabetes mellitus and hypertriglyceridaemia $(\geq 150 \mathrm{mg} / \mathrm{dL}$ or $1.7 \mathrm{mmol} / \mathrm{L}$ ), as assessed by reduction in TG and other markers of TG-rich lipoproteins. Pemafibrate treatment also lowered fasting glucose, insulin and homeostasis model assessment of insulin resistance (HOMA-IR) levels [91]. In a hyperinsulinaemic-euglycemic clamp study in subjects with hypertriglyceridaemia and insulin resistance, pemafibrate treatment improved hepatic glucose uptake and insulin sensitivity [92]. This effect may be attributed to the stimulation of fatty acid beta-oxidation and amelioration of liver dysfunction [92] and/or mediated by the effect of increases in FGF21, as shown by this and other studies [91, 92], on insulin-dependent hepatic glucose disposal [93]. These findings imply benefit with pemafibrate beyond lipid-lowering, consistent with the pharmacology of $\operatorname{PPAR} \alpha$ activation.

Pooled clinical trial data confirmed the favourable benefit-risk profile for pemafibrate. In one analysis including 1253 patients (677 also treated with a statin) with atherogenic dyslipidaemia in six phase II-III clinical trials [94•], pemafibrate $0.4 \mathrm{mg}$ daily lowered TG by $\sim 50 \%$, irrespective of statin treatment, with almost all $(98.6 \%$ on statin and $97.7 \%$ on pemafibrate monotherapy) patients showing an appropriate response. Efficacy against other components of this dyslipidaemia was robust, notably lowering remnant cholesterol by $\sim 50 \%$ (Fig. 3 ). The safety of pemafibrate was also reassuring. Regardless of statin use, pemafibrate was well tolerated, with a favourable renal and hepatic safety profile, even among patients with mild to moderate renal impairment [86]. There was no evidence of interaction with concomitant statin therapy [94•].

Finally, the PROVIDE study provided insights regarding the long-term efficacy and safety of pemafibrate $(0.2$ or $0.4 \mathrm{mg}$ daily for 52 weeks) in patients with type 2 diabetes mellitus and elevated TG [95•]. Robust lowering of TG and remnant cholesterol with pemafibrate $(\sim 50 \%)$ was sustained over this period, together with improvement in fasting insulin and HOMA-IR. Additionally, pemafibrate treatment improved liver function tests (such as alanine aminotransferase and gamma-glutamyl transferase), and was not associated with clinically meaningful increases in creatine kinase or serum creatinine, supporting the favourable safety profile of this $\operatorname{SPPARM} \alpha$.

\section{Conclusions}

SPPARM $\alpha$ poses an attractive approach to managing atherogenic dyslipidaemia associated with the metabolic syndrome. Despite early disappointment with the first compounds tested, the latest candidate, pemafibrate, has shown a promising benefit-risk profile in patients with atherogenic dyslipidaemia or hypertriglyceridaemia, key features of the metabolic syndrome. In addition to robust lowering of TG and remnant lipoproteins and elevation in HDL-C, pemafibrate treatment improved insulin sensitivity and reduced inflammation. Importantly, pemafibrate was well tolerated, with no evidence of clinically meaningful elevation in serum creatinine, a concern with conventional fibrate therapy.

The outstanding question, which has so far eluded fibrates in the setting of current evidence-based treatment including statins, is whether treatment with pemafibrate significantly reduces cardiovascular events in patients with atherogenic 
Fig. 3 Pemafibrate favourably impacts the atherogenic dyslipidaemia of the metabolic syndrome. Pooled analysis of more than 1200 patients with atherogenic dyslipidaemia showed that pemafibrate treatment for 12 weeks lowered triglycerides (TG) and associated atherogenic lipoproteins and raised HDL-C, irrespective of statin therapy. Data from Yamashita et al [94•]. apo, apolipoprotein; C, cholesterol; HDL-C, high-density lipoprotein cholesterol
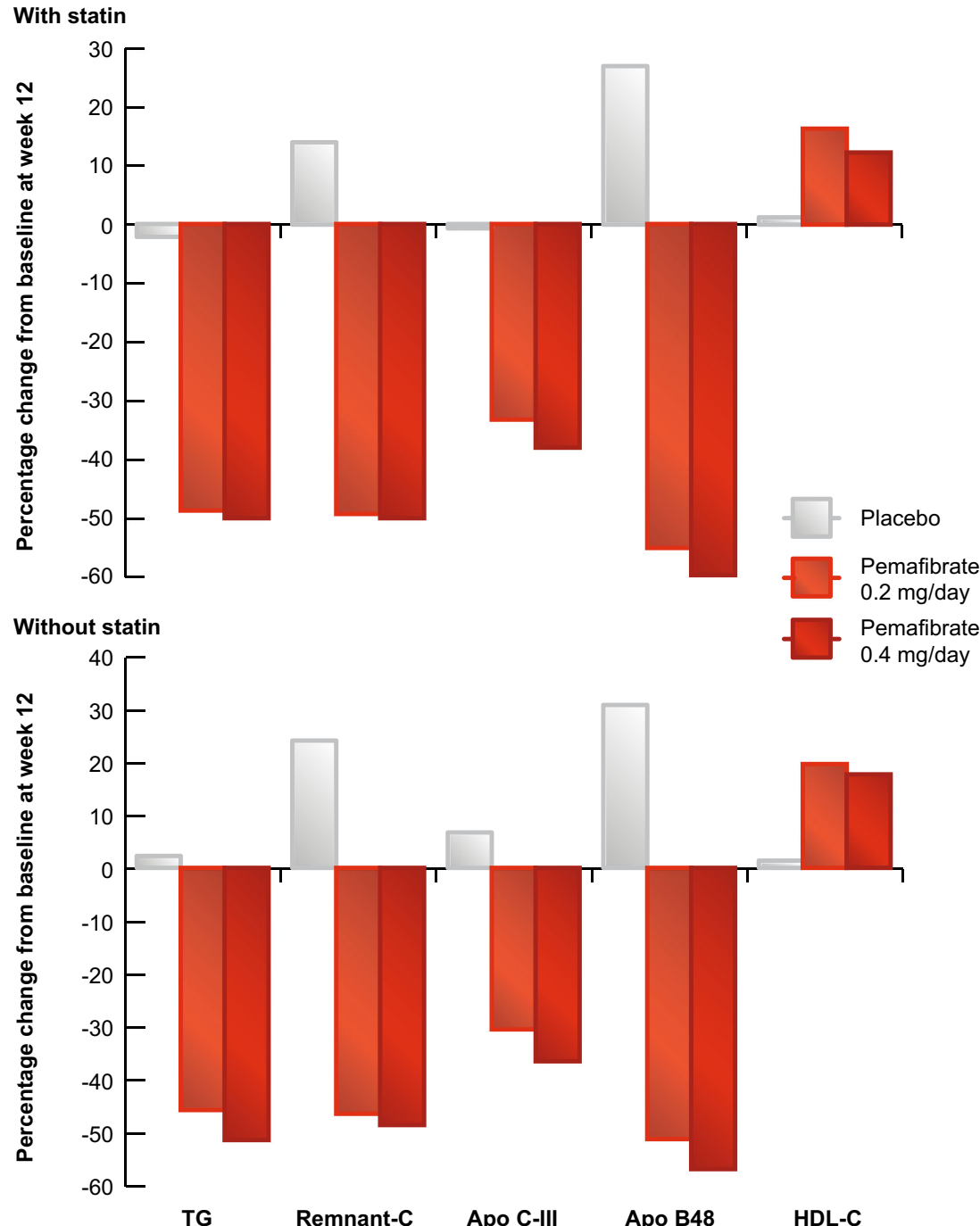

dyslipidaemia. This is being tested in the PROMINENT study (Pemafibrate to Reduce cardiovascular OutcoMes by reducing triglycerides IN diabetic patiENTs), a cardiovascular outcomes study in 10,000 patients with type 2 diabetes mellitus and atherogenic dyslipidaemia $(\mathrm{TG} \geq 2.3 \mathrm{mmol} / \mathrm{L}$ and $<5$. $6 \mathrm{mmol} / \mathrm{L}$, and low HDL-C), in both primary and secondary prevention settings [96••]. Results are eagerly anticipated in the next 2-3 years.

Finally, despite the ongoing obesity pandemic, there are still no approved treatments for NAFLD, the hepatic manifestation of the metabolic syndrome. As the SPPARM $\alpha$ pemafibrate favourably impacts lipoprotein metabolism and inflammation, it may offer therapeutic potential. Promising results in experimental NASH models and evidence of benefit in lowering liver enzymes in clinical trials are encouraging [97]. On this basis, pemafibrate is being tested in an ongoing trial in NAFLD (ClinicalTrials.gov identifier NCT03350165) [98]. It should, however, be borne in mind that the multifactorial pathogenesis of NAFLD and lack of robust surrogate trial endpoints have presented obstacles to drug development in this area.

The coming 2-3 years are critical in defining whether SPPARM $\alpha$ will offer new approaches to managing the metabolic syndrome and, as a consequence, reducing the associated morbidity, mortality and disability of ASCVD.

Authors' Contributions All authors contributed to the writing and review of this manuscript.

Funding This manuscript was prepared independently of funding.

Data Availability Data used during the current review are available from the corresponding author on reasonable request.

\section{Compliance with Ethical Standards}

Conflict of Interest J-C Fruchart and J Fruchart-Najib report personal fees from Kowa Company; MP Hermans reports no conflict of interest; T Kodama is the recipient of a research grant from Kowa Company. 
Ethics Approval Not applicable.

Human and Animal Rights and Informed Consent All reported studies/ experiments with human or animal subjects discussed by the authors have been previously published and complied with all applicable ethical standards (including the Helsinki declaration and its amendments, institutional/national research committee standards and international/national/institutional guidelines).

Consent to Participate Not applicable.

\section{Consent for Publication Not applicable.}

Open Access This article is licensed under a Creative Commons Attribution 4.0 International License, which permits use, sharing, adaptation, distribution and reproduction in any medium or format, as long as you give appropriate credit to the original author(s) and the source, provide a link to the Creative Commons licence, and indicate if changes were made. The images or other third party material in this article are included in the article's Creative Commons licence, unless indicated otherwise in a credit line to the material. If material is not included in the article's Creative Commons licence and your intended use is not permitted by statutory regulation or exceeds the permitted use, you will need to obtain permission directly from the copyright holder. To view a copy of this licence, visit http://creativecommons.org/licenses/by/4.0/.

\section{References}

Papers of particular interest, published recently, have been highlighted as:

- Of importance

•- Of major importance

1. Alberti KG, Eckel RH, Grundy SM, Zimmet PZ, Cleeman JI, Donato KA, et al. Harmonizing the metabolic syndrome: a joint interim statement of the international diabetes federation task force on epidemiology and prevention; National Heart, Lung, and Blood Institute; American Heart Association; World Heart Federation; International Atherosclerosis Society; and International Association for the Study of Obesity. Circulation. 2009;120: 1640-5.

2. O'Neill S, O'Driscoll L. Metabolic syndrome: a closer look at the growing epidemic and its associated pathologies. Obes Rev. 2015;16:1-12.

3. Azizi F, Hadaegh F, Hosseinpanah F, Mirmiran P, Amouzegar A, Abdi $\mathrm{H}$, et al. Metabolic health in the Middle East and north Africa. Lancet Diabetes Endocrinol. 2019;7:866-79.

4. The GBD. 2015 Obesity collaborators. Health effects of overweight and obesity in 195 countries over 25 years. N Engl J Med. 2017:377:13-27.

5. Nolan PB, Carrick-Ranson G, Stinear JW, Reading SA, Dalleck LC. Prevalence of metabolic syndrome and metabolic syndrome components in young adults: a pooled analysis. Prev Med. 2017;7:211-5.

6. Zhao Y, Evans MA, Allison MA, Bertoni AG, Budoff MJ, Criqui $\mathrm{MH}$, et al. Multisite atherosclerosis in subjects with metabolic syndrome and diabetes and relation to cardiovascular events: the multiethnic study of atherosclerosis. Atherosclerosis. 2019;282:202-9.

7. Lakka HM, Laaksonen DE, Lakka TA, Niskanen LK, Kumpusalo E, Tuomilehto J, et al. The metabolic syndrome and total and cardiovascular disease mortality in middle-aged men. JAMA. 2002;288:2709-16.

8. Malik S, Wong ND, Franklin SS, Kamath TV, Gilbert JL, Pio JR, et al. Impact of the metabolic syndrome on mortality from coronary heart disease, cardiovascular disease, and all causes in United States adults. Circulation. 2004;110:1245-50.

9. Younossi Z, Anstee QM, Marietti M, Hardy T, Henry L, Eslam M, et al. Global burden of NAFLD and NASH: trends, predictions, risk factors and prevention. Nat Rev Gastroenterol Hepatol. 2018;15:1120.

10. Marchisello S, Di Pino A, Scicali R, Urbano F, Piro S, Purrello F, et al. Pathophysiological, molecular and therapeutic issues of nonalcoholic fatty liver disease: an overview. Int J Mol Sci. 2019;20: 1948.

11. Caligiuri A, Gentilini A, Marra F. Molecular pathogenesis of NASH. Int J Mol Sci. 2016;17:1575.

12. Piché EM, Tchernof A, Després JP. Obesity phenotypes, diabetes, and cardiovascular diseases. Circ Res. 2020;126:1477-500 Critical review that emphasises the heterogeneity of obesity and association with cardiovascular risk.

13. Neeland IJ, Poirier P, Després JP. Cardiovascular and metabolic heterogeneity of obesity: clinical challenges and implications for management. Circulation. 2018;137:1391-406 An important overview of the metabolic basis of adiposity and need for new indices in clinical practice.

14. De Larochelliere E, Cote J, Gilbert G, Bibeau K, Ross MK, DionRoy V, et al. Visceral/epicardial adiposity in nonobese and apparently healthy young adults: association with the cardiometabolic profile. Atherosclerosis. 2014;234:23-9.

15. Rodríguez-Mortera R, Caccavello R, Garay-Sevilla ME, Gugliucci A. Higher ANGPTL3, apoC-III, and apoB48 dyslipidemia, and lower lipoprotein lipase concentrations are associated with dysfunctional visceral fat in adolescents with obesity. Clin Chim Acta. 2020;508:61-8.

16.• Neeland IJ, Ross R, Després JP, Matsuzawa Y, Yamashita S, Shai I, et al. Visceral and ectopic fat, atherosclerosis, and cardiometabolic disease: a position statement. Lancet Diabetes Endocrinol. 2019;7:715-25 Important expert consensus statement which is essential reading for evidence for visceral adiposity and ectopic fat as emerging risk factors for type 2 diabetes, atherosclerosis and cardiovascular disease.

17. Thorin-Trescases N, Thorin E. Angiopoietin-like-2: a multifaceted protein with physiological and pathophysiological properties. Expert Rev Mol Med. 2014;16:e17.

18. Thorin-Trescases N, Thorin E. High circulating levels of ANGPTL2: beyond a clinical marker of systemic inflammation. Oxidative Med Cell Longev 2017;2017:1096385.

19. Horio E, Kadomatsu T, Miyata K, Arai Y, Hosokawa K, Doi Y, et al. Role of endothelial cell-derived angptl2 in vascular inflammation leading to endothelial dysfunction and atherosclerosis progression. Arterioscler Thromb Vasc Biol. 2014;34:790-800.

20. Kim J, Lee SK, Jang YJ, Park HS, Kim JY, Hong JP, et al. Enhanced ANGPTL2 expression in adipose tissues and its association with insulin resistance in obese women. Sci Rep. 2018;8: 13976.

21. Sandesara PB, Virani SS, Fazio S, Shapiro MD. The forgotten lipids: triglycerides, remnant cholesterol, and atherosclerotic cardiovascular disease risk. Endocr Rev. 2019;40:537-57 An important review of the evidence for triglyceride-rich lipoproteins and remnants in cardiovascular disease.

22. Nordestgaard BG. Triglyceride-rich lipoproteins and atherosclerotic cardiovascular disease: new insights from epidemiology, genetics, and biology. Circ Res. 2016;118:547-63.

23. Varbo A, Benn M, Tybjærg-Hansen A, Jørgensen AB, FrikkeSchmidt R, Nordestgaard BG. Remnant cholesterol as a causal risk 
factor for ischemic heart disease. J Am Coll Cardiol. 2013;61:42736.

24. Varbo A, Benn M, Tybjærg-Hansen A, Nordestgaard BG. Elevated remnant cholesterol causes both low-grade inflammation and ischemic heart disease, whereas elevated low-density lipoprotein cholesterol causes ischemic heart disease without inflammation. Circulation. 2013;128:1298-309.

25. Kolovou GD, Watts GF, Mikhailidis DP, Pérez-Martínez P, Mora $\mathrm{S}$, Bilianou $\mathrm{H}$, et al. Postprandial hypertriglyceridaemia revisited in the era of non-fasting lipid profile testing: a 2019 Expert Panel Statement. Curr Vasc Pharmacol. 2019;17:498-514 An expert statement which reviews the evidence for postprandial hypertriglyceridaemia in prediction of cardiovascular risk.

26. LeBlanc S, Coulombe F, Bertrand OF, Bibeau K, Pibarot P, Marette A, et al. Hypertriglyceridemic waist: a simple marker of high-risk atherosclerosis features associated with excess visceral adiposity/ ectopic fat. J Am Heart Assoc. 2018;7:e008139 Important paper that presents the case for routine measurement of triglycerides and waist circumference in clinical practice.

27. Byrne A, Makadia S, Sutherland A, Miller M. Optimizing nonpharmacologic management of hypertriglyceridemia. Arch Med Res. 2017;48:483-7.

28.• Fruchart JC, Santos RD, Aguilar-Salinas C, Aikawa M, Al Rasadi $\mathrm{K}$, Amarenco $\mathrm{P}$, et al. The selective peroxisome proliferatoractivated receptor alpha modulator (SPPARM $\alpha$ ) paradigm: conceptual framework and therapeutic potential : a consensus statement from the International Atherosclerosis Society (IAS) and the Residual risk Reduction Initiative (R3i) Foundation. Cardiovasc Diabetol. 2019;18:71 Essential reading for the rationale and current evidence for SPPARMalpha.

29. Botta M, Audano M, Sahebkar A, Sirtori CR, Mitro N, Ruscica M. PPAR agonists and metabolic syndrome: an established role? Int J Mol Sci. 2018;19:1197.

30. Dubois V, Eeckhoute J, Lefebvre P, Staels B. Distinct but complementary contributions of PPAR isotypes to energy homeostasis. J Clin Investig. 2017;127:1202-14.

31. Issemann I, Green S. Activation of a member of the steroid hormone receptor superfamily by peroxisome proliferators. Nature. 1990;347:645-50.

32. Fruchart JC. Selective peroxisome proliferator-activated receptor $\alpha$ modulators (SPPARM $\alpha$ ): the next generation of peroxisome proliferator-activated receptor $\alpha$-agonists. Cardiovasc Diabetol. 2013;12:82.

33. Braissant O, Foufelle F, Scotto C, Dauça M, Wahli W. Differential expression of peroxisome proliferator-activated receptors (PPARs): tissue distribution of PPAR-alpha, -beta, and -gamma in the adult rat. Endocrinology. 1996;137:354-66.

34. Staels B, Dallongeville J, Auwerx J, Schoonjans K, Leitersdorf E, Fruchart JC. Mechanism of action of fibrates on lipid and lipoprotein metabolism. Circulation. 1998;98:2088-93.

35. Lefebvre P, Chinetti G, Fruchart JC, Staels B. Sorting out the roles of PPAR alpha in energy metabolism and vascular homeostasis. J Clin Invest. 2006;116:571-80.

36. Prieur X, Coste H, Rodriguez JC. The human apolipoprotein AV gene is regulated by peroxisome proliferator-activated receptoralpha and contains a novel farnesoid $\mathrm{X}$-activated receptor response element. J Biol Chem. 2003;278:25468-80.

37. Staels B, Vu-Dac N, Kosykh VA, Saladin R, Fruchart JC, Dallongeville J, et al. Fibrates downregulate apolipoprotein C-III expression independent of induction of peroxisomal acyl coenzyme A oxidase. A potential mechanism for the hypolipidemic action of fibrates. J Clin Invest. 1995;95:705-12.

38. Bougarne N, Weyers B, Desmet SJ, Deckers J, Ray DW, Staels B, et al. Molecular actions of PPAR $\alpha$ in lipid metabolism and inflammation. Endocr Rev. 2018;39:760-802.
39. Wahli W, Michalik L. PPARs at the crossroads of lipid signaling and inflammation. Trends Endocrinol Metab. 2012;23:351-63.

40. Shi L, Tu BP. Acetyl-CoA and the regulation of metabolism: mechanisms and consequences. Curr Opin Cell Biol. 2015;33:125-31.

41. Derosa G, Sahebkar A, Maffioli P. The role of various peroxisome proliferator-activated receptors and their ligands in clinical practice. J Cell Physiol. 2018;233:153-61.

42. Frick MH, Elo O, Haapa K, Heinonen OP, Heinsalmi P, Helo P, et al. Helsinki Heart Study: primary-prevention trial with gemfibrozil in middleaged men with dyslipidemia. Safety of treatment, changes in risk factors, and incidence of coronary heart disease. $\mathrm{N}$ Engl J Med. 1987;317:1237-45.

43. Rubins HB, Robins SJ, Collins D, Fye CL, Anderson JW, Elam $\mathrm{MB}$, et al. Gemfibrozil for the secondary prevention of coronary heart disease in men with low levels of high-density lipoprotein cholesterol. Veterans Affairs High-Density Lipoprotein Cholesterol Intervention Trial Study Group. N Engl J Med. 1999;341:410-8.

44. Keech A, Simes RJ, Barter P, Best J, Scott R, Taskinen MR, et al. Effects of long-term fenofibrate therapy on cardiovascular events in 9795 people with type 2 diabetes mellitus (the FIELD study): randomised controlled trial. Lancet. 2005;366:1849-61.

45. Ginsberg HN, Elam MB, Lovato LC, Crouse JR, Leiter LA, Linz P, et al. Effects of combination lipid therapy in type 2 diabetes mellitus. N Engl J Med. 2010;362:1563-74.

46. Bezafibrate Infarction Prevention (BIP) study. Secondary prevention by raising HDL cholesterol and reducing triglycerides in patients with coronary artery disease. Circulation. 2000;102:21-7.

47. Tenkanen L, Mänttäri M, Manninen V. Some coronary risk factors related to the insulin resistance syndrome and treatment with gemfibrozil. Experience from the Helsinki Heart Study. Circulation. 1995;92:1779-85.

48. Rubins HB, Robins SJ, Collins D, Nelson DB, Elam MB, Schaefer EJ, et al. Diabetes, plasma insulin, and cardiovascular disease: subgroup analysis from the Department of Veterans Affairs highdensity lipoprotein intervention trial (VAHIT). Arch Intern Med. 2002;162:2597-604.

49. Scott R, O'Brien R, Fulcher G, Pardy C, D'Emden M, Tse D, et al. Effects of fenofibrate treatment on cardiovascular disease risk in 9 , 795 individuals with type 2 diabetes and various components of the metabolic syndrome: the Fenofibrate Intervention and Event Lowering in Diabetes (FIELD) study. Diabetes Care. 2009;32: 493-8.

50. Sacks FM, Carey VJ, Fruchart JC. Combination lipid therapy in type 2 diabetes. N Engl J Med. 2010;363:692-4.

51. Tenenbaum A, Motro M, Fisman EZ, Tanne D, Boyko V, Behar S. Bezafibrate for the secondary prevention of myocardial infarction in patients with metabolic syndrome. Arch Intern Med. 2005;165: 1154-60.

52. Klempfner R, Erez A, Sagit B-Z, Goldenberg I, Fisman E, Kopel E, et al. Elevated triglyceride level is independently associated with increased all-cause mortality in patients with established coronary heart disease: twenty-two-year follow-up of the Bezafibrate Infarction Prevention Study and Registry. Circ Cardiovasc Qual Outcomes. 2016;9:100-8.

53. Elam MB, Ginsberg HN, Lovato LC, Corson M, Largay J, Leiter LA, et al. ACCORDION study investigators: association of fenofibrate therapy with long-term cardiovascular risk in statintreated patients with type 2 diabetes. JAMA Cardiol. 2017;2: 370-80.

54. Davis TM, Ting R, Best JD, Donoghoe MW, Drury PL, Sullivan $\mathrm{DR}$, et al. Effects of fenofibrate on renal function in patients with type 2 diabetes mellitus: the Fenofibrate Intervention and Event Lowering in Diabetes (FIELD) Study. Diabetologia. 2011;54: 280-90. 
55. Corsini A, Bellosta S, Davidson MH. Pharmacokinetic interactions between statins and fibrates. Am J Cardiol. 2005;96:44K-9K.

56. Bortolini M, Wright MB, Bopst M, Balas B. Examining the safety of PPAR agonists - current trends and future prospects. Expert Opin Drug Saf. 2013;12:65-79.

57. Ahsan W. The journey of thiazolidinediones as modulators of PPARs for the management of diabetes: a current perspective. Curr Pharm Des. 2019;25:2540-54.

58. Lincoff AM, Wolski K, Nicholls SJ, Nissen SE. Pioglitazone and risk of cardiovascular events in patients with type 2 diabetes mellitus: a meta-analysis of randomized trials. JAMA. 2007;298: 1180-8.

59. Nissen SE, Nicholls SJ, Wolski K, Nesto R, Kupfer S, Perez A, et al. Comparison of pioglitazone vs glimepiride on progression of coronary atherosclerosis in patients with type 2 diabetes: the PERISCOPE randomized controlled trial. JAMA. 2008;299: 1561-73.

60. Nicholls SJ, Tuzcu EM, Wolski K, Bayturan O, Lavoie A, Uno K, et al. Lowering the triglyceride/high-density lipoprotein cholesterol ratio is associated with the beneficial impact of pioglitazone on progression of coronary atherosclerosis in diabetic patients: insights from the PERISCOPE (Pioglitazone Effect on Regression of Intravascular Sonographic Coronary Obstruction Prospective Evaluation) study. J Am Coll Cardiol. 2011;57:153-9.

61. Kernan WN, Viscoli CM, Furie KL, Young LH, Inzucchi SE, Gorman M, et al. Pioglitazone after ischemic stroke or transient ischemic attack. N Engl J Med. 2016;374:1321-31.

62. Inzucchi SE, Viscoli CM, Young LH, Furie KL, Gorman M, Lovejoy AM, et al. Pioglitazone prevents diabetes in patients with insulin resistance and cerebrovascular disease. Diabetes Care. 2016;39:1684-92.

63. Viscoli CM, Inzucchi SE, Young LH, Insogna KL, Conwit R, Furie $\mathrm{KL}$, et al. Pioglitazone and risk for bone fracture: safety data from a randomized clinical trial. J Clin Endocrinol Metab. 2017;102:914 22.

64. Bays HE, Schwartz S, Littlejohn T, Kerzner B, Krauss RM, Karpf DB, et al. MBX-8025, A novel peroxisome proliferator receptor- $\delta$ agonist: lipid and other metabolic effects in dyslipidemic overweight patients treated with and without atorvastatin. J Clin Endocrinol Metab. 2011;96:2889-97.

65. CymaBay Therapeutics. Press release CymaBay Therapeutics Halts Clinical Development of Seladelpar. November 25, 2019. https:// www.globenewswire.com/news-release/2019/11/25/1951942/0/ en/CymaBay-Therapeutics-Halts-Clinical-Development-ofSeladelpar.html (Accessed 15 July 2020).

66. Lewis JS, Jordan VC. Selective estrogen receptor modulators (SERMs): mechanisms of anticarcinogenesis and drug resistance. Mutat Res. 2005;591:247-63.

67. Genfit. Press release. GENFIT: announces results from interim analysis of RESOLVE-IT Phase 3 trial of elafibranor in adults with NASH and fibrosis. May 11, 2020. https://ir.genfit.com/newsreleases/news-release-details/genfit-announces-results-interimanalysis-resolve-it-phase-3 (Accessed 15 July 2020).

68. Lanifibranor in patients with type 2 diabetes $\&$ nonalcoholic fatty liver disease. ClinicalTrials.gov Identifier: NCT03459079 https:// clinicaltrials.gov/ct2/show/NCT03459079 (Accessed 15 July 2020)

69. Inventiva press release. Inventiva's lanifibranor meets the primary and key secondary endpoints in the Phase IIb NATIVE clinical trial in non-alcoholic steatohepatitis (NASH). 15 June 2020. https:// inventivapharma.com/inventivas-lanifibranor-meets-the-primaryand-key-secondary-endpoints-in-the-phase-iib-native-clinical-trialin-non-alcoholic-steatohepatitis-nash/ (Accessed 15 July 2020).

70. Blair HA. Pemafibrate: first global approval. Drugs. 2017;77:1805-10.

71. Millar JS, Duffy D, Gadi R, Bloedon LT, Dunbar RL, Wolfe ML, et al. Potent and selective PPAR-alpha agonist LY518674 upregulates both ApoA-I production and catabolism in human subjects with the metabolic syndrome. Arterioscler Thromb Vasc Biol. 2009;29:140-6.

72. Nissen SE, Nicholls SJ, Wolski K, Howey DC, McErlean E, Wang $\mathrm{MD}$, et al. Effects of a potent and selective PPAR-alpha agonist in patients with atherogenic dyslipidemia or hypercholesterolemia: two randomized controlled trials. JAMA. 2007;297:1362-73.

73. Yamazaki Y, Abe K, Toma T, Nishikawa M, Ozawa H, Okuda A, et al. Design and synthesis of highly potent and selective human peroxisome proliferator-activated receptor alpha agonists. Bioorg Med Chem Lett. 2007;17:4689-93.

74. Yamamoto Y, Takei K, Arulmozhiraja S, Sladek V, Matsuo N, Han SI, et al. Molecular association model of PPAR $\alpha$ and its new specific and efficient ligand, pemafibrate: structural basis for SPPARM $\alpha$. Biochem Biophys Res Commun. 2018;499:239-45.

75. Kawasaki M, Kambe A, Yamamoto Y, Arulmozhiraja S, Ito S, Nakagawa Y, et al. Elucidation of molecular mechanism of a selective PPAR $\alpha$ modulator, pemafibrate, through combinational approaches of X-ray crystallography, thermodynamic analysis, and first-principle calculations. Int J Mol Sci. 2020. https://doi.org/10. 3390/ijms21010361 Critical study that elucidated the molecular mechanism of pemafibrate.

76. Fruchart JC. Pemafibrate (K-877), a novel selective peroxisome proliferator-activated receptor alpha modulator for management of atherogenic dyslipidaemia. Cardiovasc Diabetol. 2017;16:124.

77. Raza-Iqbal S, Tanaka T, Anai M, Inagaki T, Matsumura Y, Ikeda $\mathrm{K}$, et al. Transcriptome analysis of K-877 (a novel selective PPARalpha modulator (SPPARMalpha))-regulated genes in primary human hepatocytes and the mouse liver. J Atheroscler Thromb. 2015;22:754-72.

78. Hennuyer N, Duplan I, Paquet C, Vanhoutte J, Woitrain E, Touche $\mathrm{V}$, et al. The novel selective PPAR $\alpha$ modulator (SPPARM $\alpha$ ) pemafibrate improves dyslipidemia, enhances reverse cholesterol transport and decreases inflammation and atherosclerosis. Atherosclerosis. 2016;249:200-8.

79. Sairyo M, Kobayashi T, Masuda D, Kanno K, Zhu Y, Okada T, et al. A novel selective PPAR modulator (SPPARM), K-877 (pemafibrate), attenuates postprandial hypertriglyceridemia in mice. J Atheroscler Thromb. 2018;25:142-52.

80. Sandoval JC, Nakagawa-Toyama Y, Masuda D, Tochino Y, Nakaoka H, Kawase R, et al. Fenofibrate reduces postprandial hypertriglyceridemia in CD36 knockout mice. J Atheroscler Thromb. 2010;7:610-8.

81. Proctor SD. Intimal retention of cholesterol derived from apolipoprotein B100- and apolipoprotein B48-containing lipoproteins in carotid arteries of Watanabe heritable hyperlipidemic rabbits. Arterioscler Thromb Vasc Biol. 2003;23:1595-600.

82. Honda Y, Kessoku T, Ogawa Y, Tomeno W, Imajo K, Fujita K, et al. Pemafibrate, a novel selective peroxisome proliferatoractivated receptor alpha modulator, improves the pathogenesis in a rodent model of nonalcoholic steatohepatitis. Sci Rep. 2017;7: 42477 Experimental study that showed benefit of pemafibrate in a NASH model.

83. Sasaki Y, Asahiyama M, Tanaka T, Yamamoto S, Murakami K, Kamiya W, et al. Pemafibrate, a selective PPAR $\alpha$ modulator, prevents non-alcoholic steatohepatitis development without reducing the hepatic triglyceride content. Sci Rep. 2020;10:7818 Experimental study that investigated the mechanisms of pemafibrate in a NASH model.

84. Alkhouri N, Lawitz E, Noureddin M. Looking into the crystal ball: predicting the future challenges of fibrotic NASH treatment. Hepatol Commun. 2019;3:605-13.

85. Hounslow N, Mair S, Suganami H, Nakamura M. Pemafibrate has high bioavailability and is principally excreted via the liver. Atheroscler Suppl. 2018;32:157. 
86. Yokote K, Yamashita S, Arai H, Araki E, Suganami H, Ishibashi S, Of The K-Study Group OB. Long-term efficacy and safety of pemafibrate, a novel Selective Peroxisome Proliferator-Activated Receptor- $\alpha$ Modulator (SPPARM $\alpha$ ), in dyslipidemic patients with renal impairment. Int J Mol Sci. 2019;20(3):706.

87. Fruchart JC, Hermans MP, Fruchart-Najib J. Selective peroxisome proliferator-activated receptor alpha modulators (SPPARM $\alpha$ ): new opportunities to reduce residual cardiovascular risk in chronic kidney disease? Curr Atheroscler Rep. 2020;22:43 This review discusses the evidence for pemafibrate in the management of dyslipidaemia associated with chronic renal disease.

88. Ishibashi S, Yamashita S, Arai H, Araki E, Yokote K, Suganami H, et al. Effects of K-877, a novel selective PPAR $\alpha$ modulator $(\operatorname{SPPARM} \alpha)$, in dyslipidaemic patients: a randomized, double blind, active- and placebo-controlled, phase 2 trial. Atherosclerosis. 2016;249:36-43.

89. Yamashita S, Arai H, Yokote K, Araki E, Suganami H, Ishibashi S, et al. Effects of pemafibrate (K-877) on cholesterol efflux capacity and postprandial hyperlipidemia in patients with atherogenic dyslipidemia. J Clin Lipidol. 2018;12(5):1267-79.

90. Sairyo M, Kobayashi T, Masuda D, Kanno K, Zhu Y, Okada T, et al. A novel selective PPAR $\alpha$ modulator (SPPARM $\alpha$ ), K-877 (pemafibrate), attenuates postprandial hypertriglyceridemia in mice. J Atheroscler Thromb. 2018;25:1086.

91. Araki E, Yamashita S, Arai H, Yokote K, Satoh J, Inoguchi T, et al. Effects of pemafibrate, a novel selective PPAR $\alpha$ modulator, on lipid and glucose metabolism in patients with type 2 diabetes and hypertriglyceridemia: a randomized, double-blind, placebo-controlled, phase 3 trial. Diabetes Care. 2018;41:538-46.

92. Matsuba I, Matsuba R, Ishibashi S, Yamashita S, Arai H, Yokote K, et al. Effects of a novel selective peroxisome proliferator-activated receptor- $\alpha$ modulator, pemafibrate, on hepatic and peripheral glucose uptake in patients with hypertriglyceridemia and insulin resistance. J Diabetes Investig. 2018;9:1323-32.
93. Berglund ED, Li CY, Bina HA, Lynes SE, Michael MD, Shanafelt $\mathrm{AB}$, et al. Fibroblast growth factor 21 controls glycemia via regulation of hepatic glucose flux and insulin sensitivity. Endocrinology. 2009;150:4084-93.

94. Yamashita S, Arai H, Yokote K, Araki E, Matsushita M, Nojima T, et al. Efficacy and safety of pemafibrate, a novel selective peroxisome proliferator-activated receptor $\alpha$ modulator (SPPARM $\alpha$ ): pooled analysis of phase 2 and 3 studies in dyslipidemic patients with or without statin combination. Int J Mol Sci. 2019;20:5537 Pooled analysis of clinical trial data for pemafibrate in Japanese patients.

95. Araki E, Yamashita S, Arai H, Yokote K, Satoh J, Inoguchi T, et al. Efficacy and safety of pemafibrate in people with type 2 diabetes and elevated triglyceride levels: 52-week data from the PROVIDE study. Diabetes Obes Metab. 2019;21:1737-44 Long-term efficacy and safety data for pemafibrate in type 2 diabetes patients.

96.• Pradhan AD, Paynter NP, Everett BM, Glynn RJ, Amarenco P, Elam M, et al. Rationale and design of the pemafibrate to reduce cardiovascular outcomes by reducing triglycerides in patients with diabetes (PROMINENT) study. Am Heart J. 2018;206:8093 Critical rationale for the PROMINENT study with pemafibrate that is essential reading for the clinician.

97. Yokote K, Yamashita S, Arai H, Araki E, Suganami H, Ishibashi S. A pooled analysis of pemafibrate phase II/III clinical trials indicated significant improvement in glycemic and liver function related parameters. Atheroscler Suppl. 2018;32:154-5 [ISA2018 Abstract].

98. A study of pemafibrate in patients with nonalcoholic fatty liver disease (NAFLD). ClinicalTrials.gov Identifier: NCT03350165. https://clinicaltrials.gov/ct2/show/NCT03350165 (Accessed 15 July 2020).

Publisher's Note Springer Nature remains neutral with regard to jurisdictional claims in published maps and institutional affiliations. 